\title{
Measurement of Physiological Factor like Heart Rate using Facial Video Analysis
}

\author{
Sanam Kazi \\ Professor \\ M.H. Saboo Siddik \\ College of Engineering
}

\author{
Mubasshira Mansuri \\ Student \\ M.H. Saboo Siddik \\ College of Engineering
}

\author{
Nileshkumar Pandey \\ Student \\ M.H. Saboo Siddik \\ College of Engineering
}

\author{
Altamash Khot \\ Student \\ M.H. Saboo Siddik \\ College of Engineering
}

\begin{abstract}
The physiological parameters like heart rate, blood pressure are important indicator of human's physiological state, it uncovers the human's wellbeing. Recently, several paper reported methods to measure heart rate remotely from face videos. The methods used in those papers work correctly in stationary objects with controlled situations, but the performance degrades if the object in the video moves constantly and the illumination varies continuously. In this paper, the motive is to demonstrate that heart rate and other physiological parameters can be reliably estimated from Real Sense near- infrared images. This methods enables that heart rate estimation can be done which would be invariant to the illumination. This helps to extend its application in low-light like driving during night, etc. The method proposed not only uses the near-infrared channel which is designed originally to be hidden from users; but it also exploits the associated depth information for improved robustness to head pose. The proposed system utilizes face tracking and the PCA algorithm to counter their influences. Thus demonstrate that the method substantially outperforms all previous methods.
\end{abstract}

\section{Keywords}

Keywords Facial Analysis, Heart Rate Measurement, Physiological Parameters Measurement, PCA, GFT, SDM.

\section{INTRODUCTION}

Advances of computing and sensing technologies in last few decades have created an amazing field called computer vision, where the question is answered- how computer sense and reasons by looking into a scene. Computer vision techniques are now-a-days not only used in safety, comfort, fun and access control but also in the health monitoring and security. Computer-vision based non-contact diagnostic and monitoring systems get considerable attention in development and research of health technologies for setting up a preventive and proactive healthcare model. In addition to healthcare technologies, improved well-being of life developers initiated an era of computer vision-based recognition of biometrics and forensic investigation. Computer vision-based analysis of human facial video provides information regarding physiological parameters such as heart-beat rate, blood pressure and respiratory rate. It also provides a suitable source of HR to be used in biometrics and forensics. Face quality assessment include works which are related to the application of techniques used in acquiring high quality face sequence in real-time and alignment of face for further analysis. While measuring the physiological parameters the two main parameters i.e. heartbeat rate and physical fatigue are considered.

The motivation behind the proposed system is:-
As Heartbeat Rate (HR) is an important physiological parameter which provides information about the well-being of the human cardiovascular system in applications like medical diagnosis, rehabilitation training programs and fitness assessments. Increasing or decreasing a patient's HR beyond the norm in a fitness assessment or rehabilitation training, for example, can show how the exercise affects the trainee, and indicates whether continuing the exercise is safe. The problems of elderly people in turns demand the development of technologies for independent elderly living at private homes. Healthcare providers also have long sought the ability to automatically and continuously monitor the patients beyond the limit of the doctors concerns. Improvement of human activity and health monitoring technologies provides the notion to address such need.

\subsection{Aim}

Heartbeat Rate (HR) of a person reveals a it's health condition. Our proposed method aims to present an effective system for measuring Heart Rate from facial video captured in a more realistic environment than the testing environment of current systems. The proposed method utilizes a facial feature point tracking method by combining a 'Good Feature to Track' and a 'Supervised Descent Method' to demolish the limitations of currently available facial video based HR measuring systems. Such limitations include, e.g., unrealistic restriction of the subject's movement and artificial lighting during data capture. A face quality assessment system is also incorporated to automatically remove low quality facial frames that occur in a realistic video sequence to reduce erroneous results.

\section{EXISTING SYSTEM}

\subsection{Study of Heat Rate \& it's Impact on Human Life}

In these system for measuring of heart rate on computer controlled status one should be given, measures cardiograph in all the meetings of use of equipment, the heart rate of interactive meetings and exercise but they are also displayed may be about not control is proposed to be an effective measures in a machine. The natural approach in the heart rate measurement needs improvement in accuracy. The core values which is around $43.76-53.64 \mathrm{bpm}$ indicates the search result which would get reduced by error if measured using naturalist means. An important factor in measurement of human heart rate results in Chicago between 3 Epidemiological research association which are heart disease, death rate and coronary heart disease. Sudden deaths and death due to non communicable and reasons of all the mid old people of age group 3 test 41 - 60 years after 15 years of 123 people in 1900 - 56-year-old person gas Chicago company 
limited 41 years after the 18 year 5785 the company some research western power and 46 to 65 years of age were in Chicago average age of 5 years, Chicago heart association test item in the business of single convertible analysis of mortality rate of increase in universal non cardiovascular. Whereas analysis of use in heart control in the era of regression models of the reasons for model and mortality rate every association for the study of communicable and non- communicable reason multi-convertible cocks Regression models analysis control. Age of smoking, cholesterol blood pressure and every day and relative weights important representation in the heart of the patient sudden death rate risk factor and non anti-dumping factor for humans. Research of death in the same while one may be formed sudden death rate of heart beat research independent and sudden deaths of risk factors of organization and other non death. Keeping in mind heart rate link ages between general secondary schools and other risk cardiovascular disease. Thus there is major accuracy problem in this system. [1][2][3]

\subsection{Home-based Health Monitoring System for Aged}

Demographic and cultural changes in industrialized countries induce a an increased smaller social network and have a living by own self life[4]. These imply the necessity of automatic health monitoring and assisted living systems to be used more frequently for timely detection of health threats at home. In home monitoring technology can nowadays be used on both the aged population for monitoring life threatening situations, for detecting adverse events or health deterioration and geriatric patients. Therefore, there is a vastly growing interest in developing robust being not-noticeable that could be used anywhere home-based health monitoring application and services that can help older home dwellers to live safely and independently. However, due to the high variety of possible scenarios and situations, keeping record on health conditions of an aged individual at home may be extremely difficult.

This also includes the issues regarding to automated in-home monitoring technologies for aged people by describing-

a. Which disease or threats should be detected,

b. What data is suitable for detecting these health disease or threats, and

c. How to obtain the right data about an elderly home dweller.

The summarize result of the recently deployed monitoring approaches with a focus on automated detecting devices for monitoring health threats of aged patients who living alone at home. First, in order to give an overview of the problems at hand, briefly describe older adults, who would mostly benefit from healthcare supervision, and explain their eventual healththreats and dangerous situations, which need to be timely detected. Second, the summarize possible scenarios for monitoring an aged patients living at home and obtain similar functional requirements for monitoring applications. Third, identify the realistic state-of-the-art technological monitoring approaches, which are practically applied to aged patients, in general, and to geriatric patients, in particular. In order to uncover the majority of applicable solutions, survey the interdisciplinary fields of smart homes, telemonitoring, ambient intelligence, ambient assisted living, gero technology, and aging-in- place technology, among others. Consequently, the discussed related experimental studies and how they collect and analyze the measured data, reporting the application of sensor fusion, signal processing and machine learning techniques whenever possible, which are shown to be useful for improving the detection and identification of the situations that can threaten older adults health.

\subsection{Some Recent Non-Contact HR Measurement Applications using Facial Videos}

The recent studies proposed methodology such as Advancements in Noncontact \& Multiparameter Physiological Measurements Using a Webcam [10], Detecting Pulse from Head Motions in Video [11], Heart rate measurement based on a time-lapse image [12], Validation of heart rate extraction by using video captured on a camera system of a mobile phone [7] to estimate heartbeat rate from facial video. There are two methods which are used for unobtrusive detection of heart rate using facial videos. These methods are as follows:

Method 1 uses the facts that blood circulation causes periodic subtle facial skin color changes(observed using Photoplethysmography). These facial color-based methods, however, are not effective when one include issues like changes in illumination and the sensitivity to color noise during tracking.

Method 2 i.e. motion of the face by cardiovascular activities (observed using Ballistocardiography). The method proposed by Balakrisnan (Detecting Pulse) [11] is based on head motions in the video and the technique used extracts features from forehead by the method named Good Feature to Track (GFT). Then KLT feature tracker is used to generate motion trajectories of the selected feature points then estimate cyclic head motion frequency as the person's heart rate. But these methods have assumptions that the head should be approximately static during the facial video capture. The assumption means that there is neither internal nor external movement of the head during the video capturing phase. In the real life scenarios there includes both the motions. Thus these methods fail due to the failure of detection and tracking of the feature points in presence of the internal or external motion as well as when there is low light.

Thus the aim is to propose a solution which would overcome unrealistic assumption mentioned in the above solution.

\section{PROBLEM STATEMENT}

The approach during measuring of HR generally uses ECG system. Also some non-contact methods using facial videos can be used to measure HR. There are some limitations in the existing methods due to which it cannot be used in the real life scenarios. Thus this method which is proposed overcomes these limitations so that heart rate can be calculated in real life scenarios and thus can contribute help in the medical field applications. 


\section{PROPOSED SYSTEM}

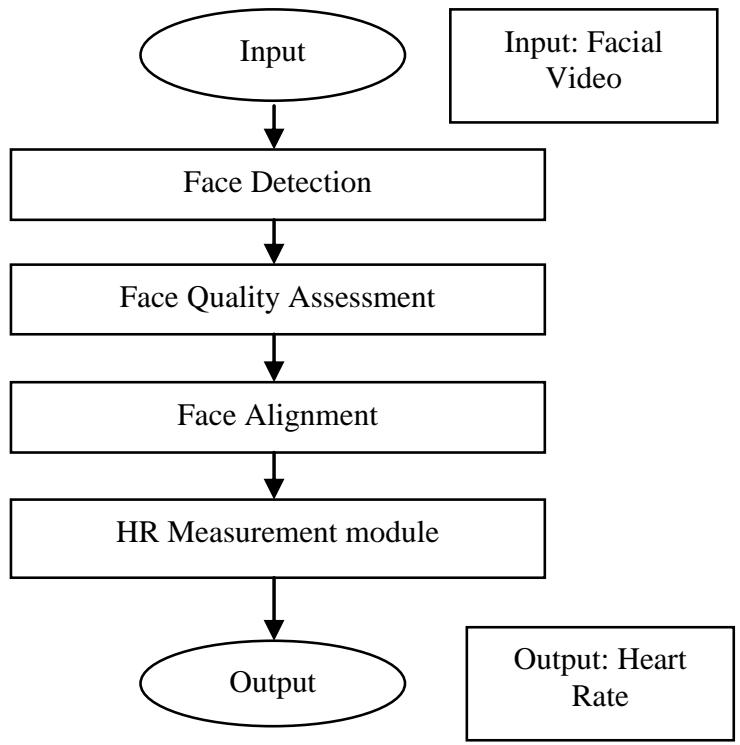

Fig 1: Flow Diagram for the steps of Proposed Solution

The proposed solution consists of multiple steps.

Step 1: Acquisition of the facial video by the camera.

In this step, appropriate Real Sense camera needs to be selected and also one should keep in mind that the video should be suitable for analysis.

Step 2: Selection of appropriate facial features.

The features that are selected should be appropriate for analysis depending on the application.

Step 3: Obtaining the outcome by using the selected features.

There exist many challenges in utilizing the appropriate images in different application. Thus the outcome is completely based on the above steps.

\subsection{Acquisition of the Quality Face Sequences in Real-Time}

The quality of the face in the video determines the performance of the solution. The quality of the face depends on a number of parameters like pose, resolution of face, sharpness and brightness. Usually a video based acquisition of images leads too many facial images for processing. Most of these images have the above mentioned problems. A Facial Quality Assessment (FQA) technique is used to process the low quality facial images to avoid the computational cost and inaccurate output and select the qualified facial images frames captured from the camera. This process reduces the amount of disqualified facial images and keeps a few best facial images which are called FACE LOG. The FAQ technique does not increases the quality of the captured facial images, however it checks whether the facial images are qualified or not. While extracting the faces from the video, the processing time is also an obstacle for achieving the real-time performance. SuperResolution image reconstruction algorithm can be a solution to the low resolution frames but it is subjected to problems like huge computational cost. Beside this improvement in the digital PTZ camera technology had lead to the availability of low cost PTZ cameras which helps in acquisition of high quality facial images in several fields.

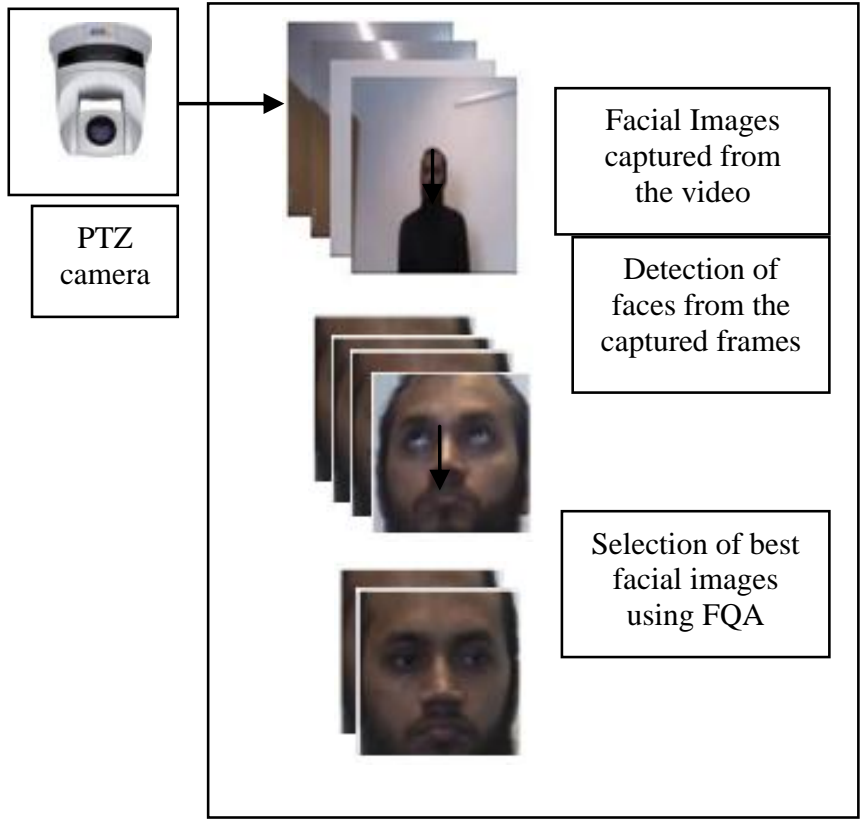

Fig 2: Procedure of Face Quality Assessment Process

The facial region of the images is considered as the Region of Interest (ROI) in the frames. The FACE LOG stores the best quality faces which are accessed on the basis of the ROI by the FQA algorithm.

\subsection{Estimation of the Facial Landmark}

Face alignment is an important step for facial image analysis. Accuracy of this step greatly depends on the above step i.e. the quality of the facial frames. If there are low quality faces it would result into erroneous alignment. Supervised Decent method (SDM) is used for face alignment along with the motion based forward extrapolation method. The face quality assessment technique measures the face quality. If the face quality is high, the system used SDM for landmark detection. If the face quality is low then motion based forward extrapolation method is used. Else if the quality is too low the landmark are discarded.

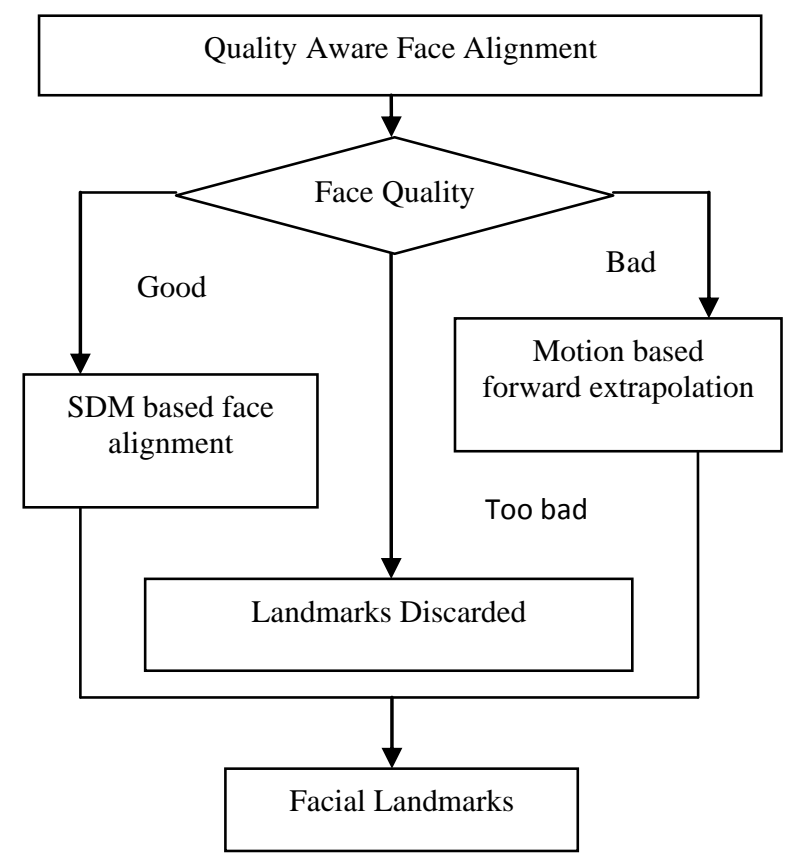

Fig 3: Block diagram for Estimation of Facial Landmark 
The SDM based face alignment system leads to the detection of erroneous landmarks in the following cases.

- Detection of face in wrong position.

- Low resolution faces.

To overcome these problems, the above procedure should be used.

\subsection{Measurement of Physiological Parameters}

The proposed method utilizes a combination of 'Good Features Track' and a 'Supervised Descent Method' for facial features point tracking. Using the above method the limitation of the existing systems used for HR measuring system using facial video can be resolved. The limitation of the existing systems is mentioned above like unrealistic restrictions of the objects movement and the lightening problem. The erroneous results are prevented by incorporating the face quality assessment technique. There is need to track the feature points of the face and develop trajectory by keep the record of head motion in the video which are caused due to heartbeat. The aim of trajectory extraction as well as signal processing is to find out the cyclic and non-cycling trajectories of the tracked points and to remove the non-cyclic components.

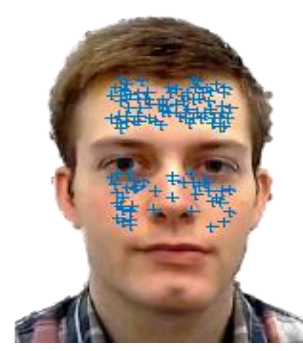

Fig 4a: Feature Points Extracted by GFT

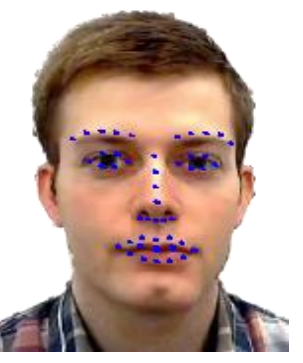

Fig 4b: Feature Points Extracted by SDM
Voluntary head motion and the facial expression changes in a video causes limitations in the GFT based tracking system. Few of these problems are listed below:
a. Erroneous tracking
b. Completely missing the track of the feature points

Thus, $80 \%$ loss of feature points in a system is observed in such cases.

While SDM does not have these limitations, thus system gets enough trajectories to measure HR. However SDM uses only 49 points when compared to GFT it uses large no of points which causes to preserve more motion information when there is no voluntary motion. Therefore the aim is to propose a method which combines the trajectories of SDM and GFT.

For generating the combined trajectories, first GFT-based tracker generates the trajectories using the face and then its result is combined with the SDM trajectories. Let the trajectories be expressed by location time-series $\mathrm{St}, \mathrm{n}(\mathrm{x}, \mathrm{y})$, where $(\mathrm{x}, \mathrm{y})$ is the location of a tracked point $\mathrm{n}$ in the video frame t.

The trajectories found in the previous step are noisy due to voluntary motion, facial expression, etc. The effect of such noise is reduced by employing the filters to the trajectories of each feature point. Human HR lie within the 0.75-5.0 Hz range thus $8^{\text {th }}$ order Butterworth band pass filter is used. These filters are applied to the HR Measurement module.
There are head motions which are generated from different sources but only those motions caused by blood circulation through aorta reflects the heat rate, thus the Principal Component Analysis algorithm (PCA) is applied to the filtered trajectories $(F)$ to separate the sources of head motion. PCA transforms $\mathrm{F}$ to a new coordinate system through calculating the orthogonal components $\mathrm{P}$ by using a load matrix $\mathrm{M}$ as follows:

$\mathrm{P}=\mathrm{S}$. $\mathrm{M}$ where $\mathrm{L}=\mathrm{M}$ is a $\mathrm{T} \times \mathrm{T}$ matrix with columns obtained from the eigenvectors of STS.

Among these separated components, the component which is most periodic is the one which belongs to the heartbeat. Then Discrete Cosine Transform (DCT) is applied to all the (P) components to find the most period one. Then employ Fast Fourier Transform (FFT) on the inverse-DCT of the component and select the first harmonic to obtain the HR. Thus the proposed method used a combination of SDM and GFT based approached.
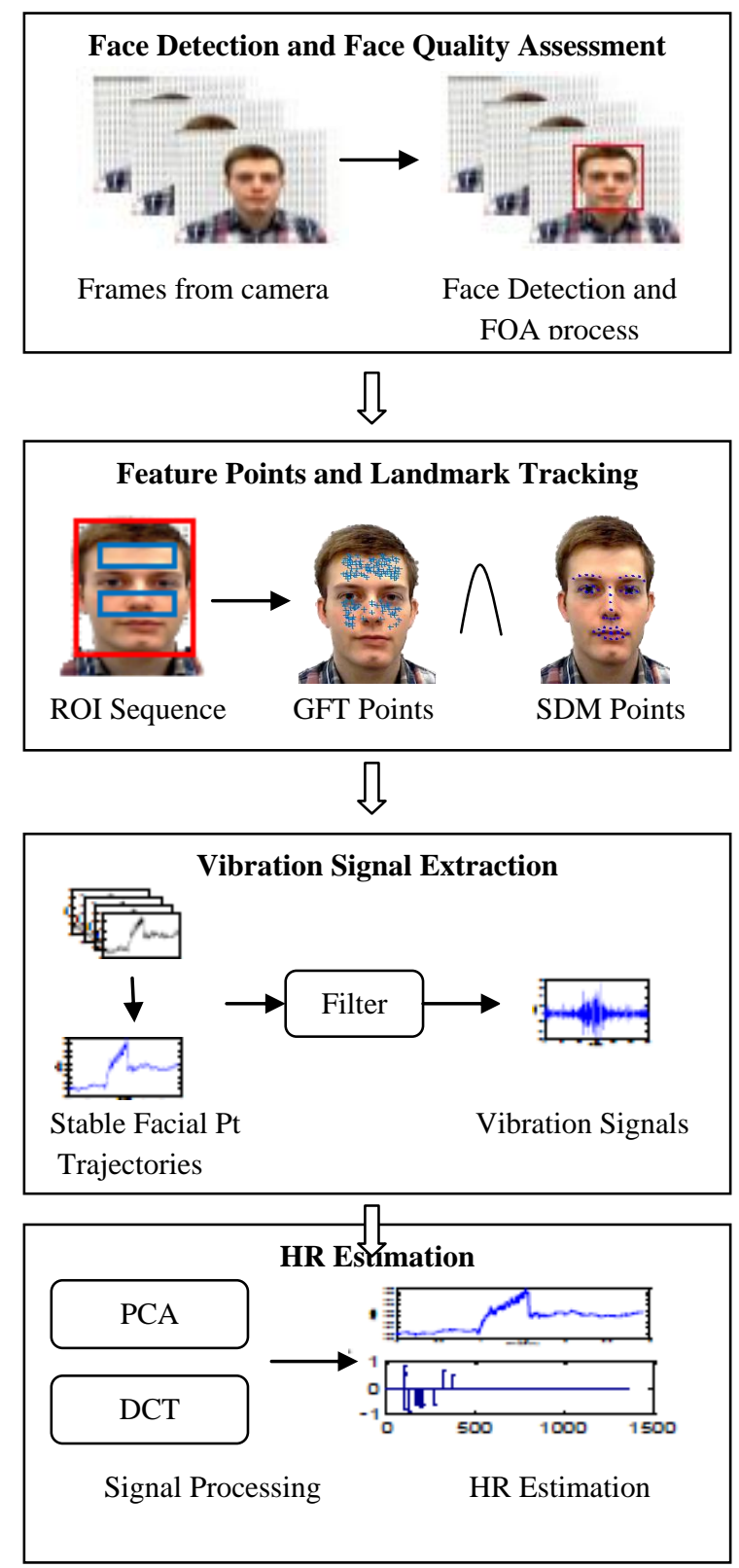

Fig 5: Block diagram for the Proposed System. 
The above figure shows the calculated average trajectories of tracked points. The trajectories are included which obtained from both GFT-based method as well as SDM-based method for facial videos with the voluntary head movement. The observation obtained from the above figure is that the GFT and SDM methods provide similar trajectories when there is very less head movement. GFT fails in case of voluntary head motion but SDM does not. Also through observations the SDM trajectories provide more comparable amplitude with the GFT trajectories, which in turn contributes to separate the heartbeat from the noise.

\section{RESULT ANALYSIS}

The comparison of the performance of this methodology against methods from [13], [5], [11], [10], [9] is done. For the purpose of comparison of accuracy measurement in terms of percentage can be used for measurement of error. The accuracy of a method is higher when there is lower error. The observations show that the solution shows consistent performance in different scenarios.

Let us consider 3 scenarios which are listed below:

Scenario 1 (normal): Subjects exposed their face in front of the cameras without any facial expression or voluntary head motion (about 60 seconds).

Scenario 2 (internal head motion): Subjects made facial expressions (sadness, surprise, laughing, angry, and fear) when looking in the cameras (about 40 seconds).

Scenario 3 (external head motion): Subjects made voluntary head movement in various directions when looking in the cameras (about 40 seconds).

By using a combination of both GFT and SDM trajectories, methodology gets more trajectories to estimate the HR pattern. On the other hand, the previous methods suffer from fewer trajectories and erroneous trajectories from the data acquired in challenging scenarios, e.g. Balakrishnan's method showed an up to $25.07 \%$ error in HR estimation from videos having facial expression change. Thus this method outperforms the previous methods in data acquisition, including all three scenarios.

Table 1. Performance Comparison between this system and the existing system

\begin{tabular}{|c|c|c|}
\hline Method & $\begin{array}{c}\text { RMSE } \\
(\mathbf{b p m})\end{array}$ & $\begin{array}{c}\text { Mean Error Rate } \\
(\mathbf{\%})\end{array}$ \\
\hline Li et al [5] & 7.62 & 6.87 \\
\hline Kwon et al [7] & 25.10 & 23.60 \\
\hline Irani et al [9] & 5.03 & 6.61 \\
\hline Balakrishnan et al [10] & End21.00 & 20.07 \\
\hline Poh et al [11] & 13.60 & 13.20 \\
\hline Poh et al [13] & 25.90 & 25 \\
\hline Proposed Method & 3.85 & 4.65 \\
\hline
\end{tabular}

Table 1 shows the comparison of HR measurement performance of the proposed method with other existing systems. The Root Mean Square Error (RMSE) in beat-perminute (bpm) and mean error rate in percentage are calculated to compare the results. From the results one can observe that
Li's [5], Irani's [9], and this method shows considerably higher results than the other existing methods as it considers the presence of voluntary head movement in the video. However, unlike Li's facial color-based method [5], Irani's method [9] and this method are motion-based methods. Thus, changing the illumination condition does not greatly affect the motion-based methods, as indicated by the results. Lastly, according to the observation this solution outperforms all the existing methods in the accuracy of HR measurement.

\section{CHALLENGES FACED}

There are some challenges faced by this system. They are as follows:

- This challenge is associated with capturing of the facial images from the video. Practically the video based images acquisition there are many facial images captured for the further process; most of those images face the problem of pose variation, low resolution, brightness issues, etc. The performance of the solution/application mainly depends on the image quality or the face quality in that image. Therefore, a quality aware method for facial acquisition should mandatorily since it is the basis necessity of the further analysis.

- The Second challenge arises in selecting the correct facial features that are need to be obtained for the further process in an application. So investigation is necessary to select the appropriate features.

- Last but not least challenge is also associated with how to use the selected feature to find the correct outcome of the application. Thus, how to utilize the feature to generate the outcome implies a challenge to be addressed.

\section{CONCLUSION}

The system measures heart rate from the video captured in realistic scenarios unlike previous existing methods which did not work well when there is voluntary motion of head, expression changes, change in lightning conditions. This system overcomes problem of all the above methods by using SDM-based method along with GFT-base method and provided good results. The HR measurement performance of this system is highly accurate and reliable in the challenging real-life environment as demonstrated in the section 6 of the paper. Thus, this methodology can be used in applications for HR measurement for real-time situations and other soft biometrics applications.

\section{FUTURE SCOPE}

The human face is not only used for biometric but also as soft biometric. The soft biometrics includes scars, gender, ethnicity, height, etc. The soft biometric are useful in application domains like healthcare, Robotics, Human Computer Interaction (HCI), medical domain, forensics and gaming. The soft biometrics is also based on physical characteristics of the human face.

The heart rate which is extracted from the facial video using non-contact ways is one of the important physiological feature of human can be used for various purpose in the computer vision applications. These can be used for improving communication skills of children suffering from Autism Spectrum Disorder in a virtual reality environment. It can also be used stress monitoring, emotion recognition applications. Based on the results, the heart rate can give promising results in the above mentioned soft biometric applications. 


\section{REFERENCES}

[1] M.c. Jones, and Paul Viola, Object detecting with cascade of simple feature, Jul. 2001

[2] Balakrishnan , Data Driven medical group, MIT Cambridge university, 2013

[3] Bruce D. Lucas, Takeo Kanade, Lucas kanade method, 1981

[4] C. Wrzus, M. Hänel, J. Wagner, and F. J. Neyer, "Social network changes and life events across the life span: A meta-analysis.," Psychol. Bull., vol. 139, no.1, pp. 53-80, 2013.

[5] X. Li, J. Chen, G. Zhao, and M. Pietikainen, "Remote Heart Rate Measurement From Face Videos Under Realistic Situations," in IEEE Conference on Computer Vision and Pattern Recognition (CVPR), 2014, pp. 43214328 .

[6] M. P. Hyett, G. B. Parker, and A. Dhall, "The Utility of Facial Analysis Algorithms in Detecting Melancholia," in Advances in Face Detection and Facial Image Analysis, M. Kawulok, M. E. Celebi, and B. Smolka, Eds. Springer International Publishing, 2016, pp. 359375 .

[7] S. Kwon, H. Kim, and K. S. Park, "Validation of heart rate extraction using video imaging on a built-in camera system of a smartphone," in 2012 Annual International Conference of the IEEE Engineering in Medicine and Biology Society (EMBC), 2012, pp. 2174-2177.

[8] S. Kwon, H. Kim, and K. S. Park, "Validation of heart rate extraction using video imaging on a built-in camera system of a smartphone," in 2012 Annual International Conference of the IEEE Engineering in Medicine and
Biology Society (EMBC), 2012, pp. 2174-2177.

[9] R. Irani, K. Nasrollahi, and T. B. Moeslund, "Improved Pulse Detection from Head Motions Using DCT," in 9th International Conference on Computer Vision Theory and Applications (VISAPP), 2014, pp. 1-8.

[10] G. Balakrishnan, F. Durand, and J. Guttag, "Detecting Pulse from Head Motions in Video," in IEEE Conference on Computer Vision and Pattern Recognition(CVPR), 2013, pp. 3430-3437.

[11] M.-Z. Poh, D. J. McDuff, and R. W. Picard, "Advancements in Noncontact, Multiparameter Physiological Measurements Using a Webcam," IEEE Trans. Biomed. Eng., vol. 58, no. 1, pp. 7-11, Jan. 2011.

[12] C. Takano and Y. Ohta, "Heart rate measurement based on a time-lapse image," Med. Eng. Phys., vol. 29, no. 8, pp. 853-857, Oct. 2007.

[13] M.-Z. Poh, D. J. McDuff, and R. W. Picard, "Noncontact, automated cardiac pulse measurements using video imaging and blind source separation," Opt.Express, vol. 18, no. 10, pp. 10762-10774, May 2010.

[14] M. Soleymani, J. Lichtenauer, T. Pun, and M. Pantic, "A Multimodal Database for Affect Recognition and Implicit Tagging," IEEE Trans. Affect. Comput., vol. 3, no. 1, pp. 42-55, Jan. 2012.

[15] J. Shi and C. Tomasi, "Good features to track," in IEEE Conference on Computer Vision and Pattern Recognition (CVPR), 1994, pp. 593-600.

[16] J. Bouguet, "Pyramidal implementation of the Lucas Kanade feature tracker," Intel Corp. Microprocess. Res. Labs, 2000. 